Noname manuscript No.

(will be inserted by the editor)

\title{
1 Moving across scales: a quantitative assessment of X-ray \\ 2 CT to measure the porosity of rocks
}

\author{
3 Ronny Pini · Claudio Madonna
}

5 Received: date / Accepted: date

Abstract We apply multidimensional X-ray CT to quantify the porosity of Berea Sandstone by using both medical- and synchrotron-based X-ray radiation, so as to produce images of the same sample with $\mathrm{mm}$ - and micron-resolution, respectively. 9 Three different samples are used and the obtained tomograms are compared by - considering the spatial distribution of porosity values for the range of voxel sizes $1 \quad 0.25-16 \mathrm{~mm}^{3}$. The agreement between the two independent techniques is assessed by means of the concordance correlation coefficient. Statistically significant correlations are found for each sample up to the maximum resolution of the medical CT scanner, i.e. for images with a voxel size of $(0.5 \times 0.5 \times 1) \mathrm{mm}^{3}$. The direct comparison of images obtained by medical- and synchrotron-based X-ray radiation has a dual benefit. First, it objectively informs the segmentation step required for the binarization of the high-resolution synchrotron images that is otherwise prone to operator bias; in this context, the applicability of the proposed workflow is demonstrated with two widely applied locally adaptive thresholding algorithms, namely the hysteresis and the watershed methods. Secondly, once this calibration has occurred, the coupling of the two techniques allows analyzing porosity heterogeneity across a range of length-scales that spans over more than eight orders of magnitudes. We anticipate that the ability to perform a true multi-scale experiment may represent the required point of departure for developing up-scaling approaches that capture the inherently complex heterogeneity of rocks.

Keywords X-ray CT · image segmentation $\cdot$ porosity heterogeneity - reservoir 27 rocks

R. Pini (corresponding author)

Department of Chemical Engineering Imperial College London, London, UK

Tel.: +44 (0)2075947518

E-mail: r.pini@imperial.ac.uk

C. Madonna

Department of Earth Sciences

ETH Zurich, Zurich, Switzerland 


\section{Introduction}

Non-invasive imaging techniques, such as X-ray Computed Tomography (X-ray $\mathrm{CT}$ ), are contributing to a novel understanding of flow processes and mechanical behavior in geomaterials $[1,2]$ and their benefits within the field of 'reservoir core analysis' were recognized since the early days [3,4]. However, the current gap existing between measurements performed at various scales (and resolutions) affects our ability to fully take advantage of this technology. In fact, with the aim of revealing the complexity of the pore space of rocks, X-ray CT is limited to a length-scale that represents a trade-off between sample size and accessible image resolution [5]. On the one hand, the small grain size of reservoir rocks requires a spatial resolution as low as a few micrometers to visualize both the geometry of the pore space and, accordingly, the curvatures of fluid-fluid interfaces; micro-CT or synchrotron-based radiation can be used to this aim on relatively small samples (typically around $6 \mathrm{~mm}$ in diameter and 5-7 $\mathrm{mm}$ in length [6-8], although the actual imaged field-of-view used for analysis rarely exceeds $2-3 \mathrm{~mm}$ in both the vertical and horizontal directions). On the other hand, whole rock cores (typically $3.5-5 \mathrm{~cm}$ in diameter and at least $10 \mathrm{~cm}$ in length) are used to provide key parameters for reservoir formation evaluation, such as relative permeability and capillary pressure curves [9-12]; in those studies a medical X-ray CT scanner is used to image the samples, though at the expense of a lower resolution (a few $\mathrm{mm}$ and above for quantitative analyses [13]). It follows that any parameter derived from experiments performed on whole cores is inherently effective, as it incorporates the effects of structural and chemical heterogeneities at finer scales and observed in micro-scale studies. At the same time, highly-resolved observations of flows on samples of a few $\mathrm{mm}^{3}$ cannot capture the complexities introduced by the presence of larger $(\mathrm{mm}-\mathrm{cm})$ sub-core scale features that are characteristic of sedimentary rocks [14-16] and whose effects are commonly observed during multiphase flows with whole rock cores [17-21]. In the attempt of upscaling rock properties measured on core samples for their use in reservoir flow simulations, the concept of missing-scale is adopted when referring to the assumption that an original data-set is representative of a much larger volume and sub-volume heterogeneity is ignored [22]. We argue that the same concept describes well the current knowledge gap in laboratory practices for reservoir core analysis. In fact, only the ability to directly associate pore-scale structures with sub-core scale patterns would pave the way towards a holistic approach to the study of the role of heterogeneity on fluid transport in rocks and other porous media.

Since no single technique currently exists that enables non-invasive measurements across the full range of relevant length-scales (micron to $\mathrm{cm}$ in this paper), protocols are needed that bridge the gap between these observations. Studies exploiting $\mathrm{X}$-ray CT for a true multi-scale analysis of rock properties are just beginning and entail practices, such as the physical sub-sampling for high-resolution imaging and the registration of the obtained images within the original core sample to integrate information at different length-scales [23-25]. Efforts are also directed towards the establishment of robust methods to reduce the inherent noise associated with the reconstructed X-ray tomograms. In fact, despite the extensive experience with medical CT scanning, protocols are still being revisited to enable the quantitative estimation of porosity and fluid saturation in opaque porous media down to resolutions of only a few $\mathrm{mm}^{3}[13,26]$. As summarized in the next section, efforts 
are still underway in the use of micro $\mathrm{CT}$ or synchrotron data to reach a level of standardization comparable to medical CT scanning [5]. As a matter of fact, the latter can benefit from studies where results obtained from the two independent techniques are compared, e.g. by imaging the same sample at increasingly higher resolutions.

As a first step in this direction, we present results from an experimental campaign involving the use of medical X-ray CT and synchrotron-based X-ray radiation to image and quantify the porosity of Berea Sandstone samples. The novelty of the work is that experiments have been carried out such that a direct comparison is made between results obtained from the two independent techniques. Statistical measures are used to evaluate the quantitative agreement between the average and spatially distributed porosity values at various voxel sizes $\left(0.25-16 \mathrm{~mm}^{3}\right)$. A practical implication of this study is that a protocol has been established that exploits the comparison between the two techniques to objectively inform the segmentation process required in the analysis of micro-CT tomograms.

\section{Quantifying porosity from X-ray tomograms}

Porosity represents one of the primary variables that can be obtained from the analysis of X-ray tomograms of a rock sample and the resolution of the acquired images determines the type of approach for its computation. When the voxel size is much larger than the characteristic pore size of the rock under study (such as for medical X-ray CT), the so-called X-ray saturation technique is used, where scans of the core saturated with two different fluids (typically air and water) are properly combined [27]:

$$
\phi_{i}=\frac{C T_{\mathrm{wr}, i}-C T_{\mathrm{ar}, i}}{C T_{\mathrm{w}}-C T_{\mathrm{a}}}
$$

where $\phi_{i}$ are voxel porosities, $C T_{\mathrm{wr}, i}$ and $C T_{\mathrm{ar}, i}$ are the CT numbers in Hounsfield units assigned to each voxel $i$ when the core is saturated with water and air, respectively, and $C T_{\mathrm{w}}$ and $C T_{\mathrm{a}}$ are the corresponding (constant) values of the pure fluids. This method (and equation) for computing the porosity of rocks was proposed by [4], where a direct comparison with independent measurements was carried out, and has since then been applied to a wide range of rock types $[18,28$, $9,12,29,30]$. Note that Eq.1 was originally derived in terms of linear attenuation coefficients $\mu$, i.e. the truly measurable quantity by X-ray absorption [4], and uses $C T \propto\left(\mu-\mu_{\mathrm{w}}\right) / \mu_{\mathrm{w}}[27]$. Most importantly, Eq.1 implies that the CT number of a voxel can be expressed as the linear combination of the CT numbers associated with the volume fractions of each of its components, e.g. $C T_{\mathrm{wr}}=\phi C T_{\mathrm{w}}+(1-\phi) C T_{\mathrm{r}}$; given that mass is an extensive property, this assumption is justified because the linear attenuation coefficient (and, accordingly, the CT number) is proportional only to bulk density for X-ray energies above $100 \mathrm{keV}$, i.e. where medical CT scanners normally operate $[3,31]$. The main advantage of the X-ray saturation technique is that the use of two different fluids allows eliminating the CT number associated with the rock matrix $\left(C T_{\mathrm{r}}\right)$; the latter is in fact not known a priori when heterogeneous materials, such as rocks, are studied and where it can additionally vary spatially. Porosity heterogeneity at the voxel level can be readily quantified from Eq.1 upon reduction of the noise that affects X-ray images; the 
latter can easily produce a relative error of $10-15 \%$ in the computed porosity for $(0.5 \times 0.5 \times 1) \mathrm{mm}^{3}$ voxels [13], i.e. an uncertainty that is similar in strength to spatial variations in the porosity of many reservoir rocks at the same scale [32, 33,17-19]. As explained in a previous publication [13], this uncertainty can be assessed by subtracting two scans that have been taken at the same position and it can be significantly reduced by proper combination of sufficient repeated measurements and image coarsening (see Section 3.2).

When highly resolved images are available (such as for synchrotron-based X-ray radiation or benchtop micro-CT scanners), the approach is preferred of a segmentation that partitions the image into pore space and mineral matrix [1]. A proper segmentation of the image is of paramount importance because the obtained binarized representation of the given sample (i) enables a straightforward computation of the porosity and (ii) represents the framework for the computation of other rock properties (e.g., permeability, capillary pressure and elastic moduli) [34,35]. It should be noted that the segmentation step can be preceded by the application of noise-reduction filters, although the good quality of the images obtained from synchrotron sources often allows for direct processing [2]. Ideally, the segmentation step is carried out by means of an automated (i.e. unsupervised) algorithm, so as to save time, eliminate operator bias and produce an objectively reliable data-set. The reality is that such algorithm does not exist [34], thus requiring some degree of manual interaction and quality control, especially when different types of porous media are considered $[1,35]$. The direct consequence of this is that the application of the various available segmentation methods leads to as many different results, as summarized in a number of studies where direct comparisons have been made using both artificial and natural unconsolidated porous materials $[34,36]$ as well as intact rocks [35,37]. As expected, these variations were attributed to both the choice of the method and to the operator bias in setting the threshold intensity needed to distinguish between pore space and solid phase. For instance, relative errors up to $13 \%$ and $31 \%$ were observed when different algorithms were used to compute the porosity of the exact same cuboid of a Berea Sandstone and a carbonate sample, respectively [35]. Or, relative errors of $1-8 \%$ in the computed porosity were found upon varying the threshold intensity of $\pm 2 \%$ for three different segmentation approaches applied to a Berea Sandstone sample [37]; interestingly, much larger deviations were observed in the same study for the permeabilities computed on the same sample (8-30\%). Unfortunately, the same groups of authors agree that no definitive conclusion can be drawn from their studies due to the lack of an absolute reference that would define the optimal binarization result $[34,36,37]$. In this context, it has been suggested that any study using results from image segmentation should include a sensitivity analysis associated with the value of the threshold intensity and/or the benchmarking with independent measurements $[36,38,37]$. With regards to the latter, it is worth noting that the small size of the volume that is typically imaged in micro-CT or synchrotron studies makes a one-to-one comparison with experimental results a very challenging task. As anticipated above, in the present study we attempt resolving both issues by performing a direct comparison between porosity values computed from synchrotronand medical-CT images. 


\section{Methodology}

Figure 1 illustrates the workflow of the multi-scale imaging experiment presented in this study, which includes (i) the imaging of a large $(5 \mathrm{~cm}$-diameter and $10 \mathrm{~cm}$ long) cylindrical rock sample using a medical X-ray CT scanner, followed by (ii) the coring of three smaller (6 mm-diameter and $10 \mathrm{~mm}$-long) plugs at specific locations within the inlet face of the larger sample, (iii) the subsequent imaging of the plugs using synchrotron-based radiation, and (iv) the comparison of the reconstructed tomograms, i.e. rectangular cuboids with size $(4 \times 4 \times 1) \mathrm{mm}^{3}$, in terms of their average and spatially distributed porosity at various resolutions. A more detailed description of each step and of the rock sample used in the experiment is given below. The abbreviations $\mathrm{mCT}$ and $\mu \mathrm{CT}$ will be used throughout the text when referring to images obtained using the medical X-ray $\mathrm{CT}$ and the synchrotronbased X-ray method, respectively.

\subsection{Materials}

A Berea Sandstone core (Cleveland Quarries, Ohio USA) was used in this study. The sample is a well-sorted sandstone (grain size 100-200 $\mu \mathrm{m}$ ) dominated by quartz with minor presence of iron oxide and has an average porosity of $20 \%$ and a permeability of about $300 \mathrm{mD}$. Prior to the start of the experimental campaign, the sample was fired at $700^{\circ} \mathrm{C}$ for $2 \mathrm{hrs}$. to stabilize swelling clays. The sample is a relatively homogeneous sandstone that however contains finer-textured strata that are not readily visible in the dry or fully saturated state $[21,14]$ and that are associated with fine-scale $(\mathrm{mm}-\mathrm{cm})$ variations in the porosity (about $10 \%$ rel. for this specific sample). Also, Berea Sandstone is widely used as a proxy for reservoir rocks, thus making it an ideal candidate to validate the protocols developed in this study.

\subsection{Medical X-ray CT scanner (mCT)}

A medical X-ray CT scanning instrument (General Electric Hi-Speed CT/i Xray computed tomography) was used to obtain multidimensional porosity maps of the large core sample. The following imaging parameters were applied: a voxel dimension of $(0.5 \times 0.5 \times 1) \mathrm{mm}^{3}$, a tube current of $200 \mathrm{~mA}$, an energy level of the radiation of $120 \mathrm{keV}$ and a display field of view of $25 \mathrm{~cm}$. For image reconstruction, a 'body filter' and a 'standard' convolution kernel were used, both provided with the instrument's software package. Voxel porosities $\phi_{i}$ are obtained by applying the X-ray saturation technique, Eq.1. In the equation, the CT number of pure water $\left(C T_{\mathrm{w}}=0\right)$ is known from a previous calibration with a phantom, while the CT number of air $\left(C T_{\mathrm{a}}=-986 \mathrm{HU}\right)$ has been estimated by measuring the attenuation in the line that conducts fluids into the rock core. As reported in a previous study where the same sample was used, X-ray CT computed average porosity values are in good agreement with results obtained from a combination of Helium pycnometry and mercury intrusion porosimetry [11]. For the imaging experiment, the core was mounted in a cylindrical aluminum core-holder, so as to minimise the known phenomenon of beam-hardening [27]; the images shown 
in Figure 2 confirm the absence of a lighter shading along the perimeter of the core that is otherwise considered symptomatic of this effect. Additional details regarding the core-flooding apparatus are given in a previous publication together with the description of the procedure followed to saturate the sample with water prior to the scans [13]. Moreover, a careful evaluation of the noise affecting the CT images has been carried out and it is summarised in the Appendix; briefly, the outcome of this analysis shows that the noise is random in nature and that it can be predicted (and, accordingly, reduced) upon application of classic rules of error propagation based on the variances of normal density distribution curves. In this study, we have worked on images obtained from the average of 20 repeated $1 \mathrm{~mm}$ thick scans of the inlet face of the sample, this being the same location where small sub-samples have been subsequently cored. As shown in Figure 2, the averaging of 20 independent scans enables reducing the absolute uncertainty associated with the computed porosity at the voxel scale down to $\delta \phi=0.6 \%$, while maintaining the original voxel size of $(0.5 \times 0.5 \times 1) \mathrm{mm}^{3}$; an increase of the voxel size, $V_{\mathrm{vox}}$, to $(1 \times 1 \times 1)$ and $(2 \times 2 \times 1) \mathrm{mm}^{3}$ further reduces the error in the porosity to $\delta \phi=0.5$ and $0.3 \%$ abs., respectively. Accordingly, images (c), (d) and (e) in Figure 2 have been used for comparison with results from $\mu \mathrm{CT}$ (see Section 3.4).

\subsection{Synchrotron-based X-ray radiation $(\mu \mathrm{CT})$}

As shown in Figure 1, three 6 mm-plugs (P1, P2 and P3) have been cored from the mother sample for high-resolution imaging using synchrotron-based X-ray radiation. To this aim, the dry samples were imaged with the Advanced Light Source (ALS) at the Lawrence Berkeley National Laboratory (Berkeley, USA). For imaging, a PCO 4000 X-ray camera was used with a 5X Mitutoyo lens by keeping the sample-detector distance at $75.4 \mathrm{~mm}$. All scans were performed at a monochromatic energy level of $28 \mathrm{keV}$ by capturing 1441 projections with a resulting computed pixel dimension of $1.791 \mu \mathrm{m} /$ pixel and a spatial resolution of $1.791 \times 2 \approx$ $3.6 \mu \mathrm{m}$ (as suggested by the Nyquist-Shannon theorem [39]). The acquired tomograms were first cropped to rectangular cuboids with size $(4 \times 4 \times 2) \mathrm{mm}^{3}$. The digital image processing was carried out using AVIZO Fire 8.0 (FEI Visualization Sciences Group); a close-up figure $\left(1 \times 1 \mathrm{~mm}^{2}\right)$ of a synchrotron tomogram is shown in Figure 3 to provide an overview of the steps from the raw (A), to the filtered (B) and segmented images (hysteresis, C, or watershed, D). In particular, a socalled 'non-local mean denoising' algorithm (NLM) was first employed to reduce image noise [40] with the following parameters: a search-window of $21 \times 21$ pixels, a local neighborhood of $5 \times 5$ pixels and a similarity value of 0.4 . The obtained 8 -bit gray-level $\mu \mathrm{CT}$ images were then binarized by means of the bilevel (or hysteresis) thresholding algorithm [41]. The latter requires the identification of two threshold levels $\left(T_{\min }\right.$ and $\left.T_{\max }\right)$ that are used to define two classes of objects: the lower class (class $s_{0}$, representing the pore space) that includes pixels with intensity lower than $T_{\text {min }}$ and the upper class (class 1 , representing the rock's skeleton) that includes pixels with intensity greater than $T_{\max }$. Additionally, any pixel belonging to class $_{0}$ acts as seed point for a so-called 'region-growing image segmentation' algorithm, which adds to the lower class pixels found in the local neighborhood, while the remaining pixels are added to class $_{1}$. In this study, the value of $T_{\max }$ is set as being the mode of the grey-level intensity histogram of the image; as explained below, 
$T_{\min }$ is used as a parameter to match the mCT porosity values. As an example of general validity, the histogram representing the grey-level intensity of a tomogram after denoising is shown in Figure 4 . While two distinct peaks are readily recognizable in the histogram, they also delimit a relatively wide valley (with intensity level 35-43), thus making the selection of the appropriate value for $T_{\min }$ not trivial. Accordingly, the bins with a brighter color have been used to quantify the effects of $T_{\min }$ on the computed porosity. For a given cuboid, the latter is calculated from the $2 \mathrm{D}$ images as,

$$
\phi=\frac{1}{N} \sum_{n=1}^{N} \frac{\text { class }_{0}}{\text { class }_{0}+\text { class }_{1}}
$$

where $N$ is the number of slices in the cuboid, while class $_{0}$ and class $_{1}$ represent the number of pixels attributed to the pore and solid phase, respectively. Note that $\phi$ includes both connected and non-connected porosity; for Berea Sandstone it is plausible to assume that the volume fraction of the latter is negligible, thus enabling a direct comparison with mCT-derived porosities.

In order to test the general validity of the proposed workflow, the exercise described above was repeated while applying a different thresholding algorithm that is widely used in the geosciences, namely watershed segmentation [42]. Like the hysteresis method, the watershed method belongs to the family of so-called locally adaptive algorithms and requires the user to set two thresholds $\left(T_{\min }\right.$ and $\left.T_{\max }\right)$, in addition to the gradient level that is used to demarcate class borders locally [43]. In this study, the gradient level was (arbitrarily) set to a value of $20, T_{\max }$ to a value of 55 , and $T_{\min }$ was used as a fitting parameter (see again Figure 4); porosity is then calculated using Eq.2.

It is worth highlighting that, as suggested in [44] and regardless of the algorithm used, the thickness of the CT images is kept at a minimum $(1.791 \mu \mathrm{m}$ in this study) when segmenting the images, and any coarsening (for comparison with mCT images) is applied only after this operation, so as to avoid introducing socalled partial volume effects. Also, thanks to the selected sample-detector distance, the effects caused by phase-contrast (and manifested by the presence of bright rims at the grain side of the grain/pore boundary in the close-up images shown Figure 3) are minor, thus enabling a proper segmentation of the pore space.

\subsection{Integrated analysis}

The final step in the analysis involves the comparison of the images of the same sample that have been obtained using the two X-ray methods. To this aim, the rectangular cuboids need to be properly registered, so as to ensure that porosity values are compared at the same locations, and have to possess the same voxel size with a minimum value of $(0.5 \times 0.5 \times 1) \mathrm{mm}^{3}$, this being the highest resolution achievable with the mCT images. In this study, the comparison is performed at four distinct voxel sizes, namely $V_{\mathrm{vox}}=(0.5 \times 0.5 \times 1),(1 \times 1 \times 1),(2 \times 2 \times 1)$ and $(4 \times 4 \times 1) \mathrm{mm}^{3}$, thus corresponding to $64(=8 \times 8), 16(=4 \times 4), 4(=2 \times 2)$ and 1 voxels per image, respectively.

The following approach was adopted to account for the uncertainty introduced from the physical sub-sampling (coring) of the smaller plugs when registering the 
location of each set of images: the rectangular cuboid from the $\mathrm{mCT}$ images is allowed for a translational freedom in the $(x, y)$ plane of $\pm 1 \mathrm{~mm}$ (corresponding to 25 possibilities per sample), and each of these images is compared to a $1 \mathrm{~mm}$-thick section extracted from the $(4 \times 4 \times 2) \mathrm{mm}^{3}$ volume of the $\mu \mathrm{CT}$ image. This exercise is repeated for nine different values of threshold level $\left(T_{\min }=35,37-41,43,45,47\right)$ used for segmenting the $\mu \mathrm{CT}$ images by means of hysteresis thresholding, for a total of $25 \times 558 \times 9=125,550$ comparisons per sample $(25 \times 558 \times 3=41,850$ for watershed thresholding).

The statistical significance of the correlation between porosity values obtained from $\mathrm{mCT}$ and $\mu \mathrm{CT}$ images is evaluated by means of the concordance correlation coefficient, $R_{\mathrm{C}}$, defined as [45]:

$$
R_{\mathrm{C}}=\frac{2 \sigma_{u v}}{\sigma_{u}^{2}+\sigma_{v}^{2}+(\bar{u}-\bar{v})^{2}}
$$

where $u$ and $v$ refer to the porosity values obtained from $\mathrm{mCT}$ and $\mu \mathrm{CT}$ images, respectively, $\bar{u}$ and $\bar{v}$ are the mean values of each data-set, $\sigma_{u}^{2}$ and $\sigma_{v}^{2}$ their variances, and $\sigma_{u v}$ the covariance. The latter have been calculated using the 'mean', 'var' and 'covar' functions in MATLAB with the normalization factor set equal to the number of observations. Note that the concordance correlation coefficient specifically measures how close the observed data are to the identity line $v=u$, this being particularly appropriate when evaluating the strength of agreement between two independent methods (as opposed to a simple measure of linear correlation given by the Pearson coefficient). Accordingly, $R_{\mathrm{C}}$ can vary between 0 ( $v$ and $u$ are uncorrelated) and 1 (perfect agreement). In this study, the correlation is called significant when $R_{\mathrm{C}} \geq R_{\mathrm{C}}^{0}=0.95,0.5$ and 0.25 for the $(2 \times 2),(4 \times 4)$ and $(8 \times 8)$ schemes, respectively, and the best fit (i.e. the location, and, accordingly, the value for $\left.T_{\min }\right)$ is associated with the maximum value of the mean of the correlation coefficients found for the three schemes. Note that the threshold values $R_{\mathrm{C}}^{0}$ have been selected based on $\operatorname{Prob}_{N}\left(R_{\mathrm{C}} \geq R_{\mathrm{C}}^{0}\right) \leq 5 \%$, i.e. a correlation is called significant if the probability is less than $5 \%$ that $N$ measurements of two sets of uncorrelated porosity values (mCT and $\mu \mathrm{CT}$ ) would give a coefficient $R_{\mathrm{C}}$ larger than $R_{\mathrm{C}}^{0}$. As reported in [46], the latter takes a value of $0.95,0.5$ and 0.25 for $N=4,16$ and 64 , respectively.

\section{Results}

In Table 1 are summarized for each sample the average porosity values that have been obtained from the $\mathrm{mCT}$ and $\mu \mathrm{CT}$ images, together with the values of the concordance correlation coefficient, $R_{\mathrm{C}}$, used to assess the agreement between the two techniques at different spatial resolutions of the scans. The latter correspond to the following voxel sizes: $(2 \times 2 \times 1),(1 \times 1 \times 1)$ and $(0.5 \times 0.5 \times 1) \mathrm{mm}^{3}$, and are referred to as the $(2 \times 2),(4 \times 4)$ and $(8 \times 8)$ schemes, respectively. It can be concluded from the values reported in the table that (i) an excellent agreement is obtained between mCT- and $\mu \mathrm{CT}$-derived porosities (with deviations less than $0.1 \%$ abs. for a whole sample of $\sim 16 \mathrm{~mm}^{3}$ ) and that (ii) this agreement includes the spatial distribution of the porosity values within a given sample down to a voxel size of $(0.5 \times 0.5 \times 1) \mathrm{mm}^{3}$. In fact, statistically significant correlations are 
achieved between the two techniques, as reflected by the large values of the $R_{\mathrm{C}}$ coefficient. It is worth noting that while for the $(4 \times 4)$ and $(8 \times 8)$ schemes $R_{\mathrm{C}}$ is indeed always greater than $R_{\mathrm{C}}^{0}$, the opposite is true for the coarser $(2 \times 2)$ scheme $\left(R_{\mathrm{C}}<R_{\mathrm{C}}^{0}=0.95\right)$. The reason for this may be found in the similarity between the spatial variability of the porosity at this scale $\left(\sim 0.5 \%\right.$ abs. for $4 \mathrm{~mm}^{3}$ voxels $)$ and the uncertainty affecting the $\mathrm{mCT}$ measurements at the same scale $(\sim 0.3 \%$ abs.). Nevertheless, the fact that $R_{\mathrm{C}}$ values of 0.7 and above are found for the $(2 \times 2)$ scheme is a firm indicator of a strong correlation.

The excellent agreement achieved between $\mathrm{mCT}$ - and $\mu \mathrm{CT}$-derived porosities can be better appreciated by looking at Figures 5 and 6 , where for each sample the spatially distributed porosity values are plotted for the $2 \times 2$ (i.e. $V_{\mathrm{vox}}=4 \mathrm{~mm}^{3}$ ), and $4 \times 4$ (i.e. $V_{\text {vox }}=1 \mathrm{~mm}^{3}$ ) schemes, respectively. In each plot, the black-filled symbols represent voxel porosity values obtained from $\mathrm{mCT}$ images, while the empty symbols are $\mu \mathrm{CT}$ results that correspond to the optimum value of threshold level $\left(T_{\mathrm{min}}^{\mathrm{o}}\right)$ used for image segmentation using hysteresis thresholding. The black lines represent also $\mu \mathrm{CT}$ data, but obtained upon varying $T_{\min }$. Pairs of $2 \mathrm{D}$ colored porosity maps are additionally shown in each plot to visually support the quantitative agreement between the two imaging methods. The detrimental effect of varying $T_{\min }$ on the agreement between $\mathrm{mCT}$ - and $\mu \mathrm{CT}$-derived porosities represents the second striking feature to be appreciated from these plots. Interestingly, the relative error introduced is clearly visible for larger voxel sizes (Figure 5), while it decreases with increasing image resolution (Figure 6). This behavior is associated with the simultaneous increase of the strength of porosity heterogeneity with decreasing voxel size. Nevertheless, the discrepancy caused on both average and voxel-computed porosities is significant and larger than the uncertainty affecting the $\mathrm{mCT}$ results for the range of $T_{\min }$ values studied. Moreover, the optimum value for $T_{\min }$ is not unique, but changes from sample to sample, thus supporting the adoption of the approach presented here that exploits an independent measurement for defining the optimal binarization of the given image. We anticipate here that for the range of $T_{\min }$ values considered here, differences in the obtained images could be hardly assessed without a quantitative measure, such as the one based on the concordance correlation coefficient presented in this study.

Correlation diagrams are shown in Figure 7, where $\mu \mathrm{CT}$ - and mCT-porosity values are plotted against each other for all samples and for the three schemes of voxel-sizes $V_{\text {vox }}$, namely $4 \mathrm{~mm}^{3}(2 \times 2), 1 \mathrm{~mm}^{3}(4 \times 4)$ and $0.25 \mathrm{~mm}^{3}(8 \times 8)$. In each plot, the black filled symbols are the results from $\mu \mathrm{CT}$ images using the optimum threshold level ( $T_{\min }^{\mathrm{o}}$, hysteresis thresholding), while for the grey-filled and empty symbols $T_{\min }=T_{\min }^{\mathrm{O}} \pm 4$. In the plots, the dashed line represents a perfect correlation (identity line, $R_{\mathrm{C}}=1$ ). The first two diagrams from the left reflect most of the conclusions drawn earlier, with the very good agreement between the two techniques (readily noticeable by the gathering of the symbols in the very vicinity of the identity line) and the pronounced effect of the selected level of thresholding on the calculated porosity values. Most importantly, the agreement between the two imaging methods is observed over the whole range of values that typically characterizes the spatial variability of the porosity at this scale $\left(\phi_{i}=18-22 \%\right)$. The agreement between the two techniques observed in the correlation diagram prepared for the smallest voxel size $\left(V_{\text {vox }}=0.25 \mathrm{~mm}^{3}\right.$, third plot from the left) is less obvious, as evidenced also by the pair of $2 \mathrm{D}$ porosity maps shown in the bottomright corner of the figure. In particular, it is noticeable how the $\mu \mathrm{CT}$-porosity 
values show a wider range of porosity heterogeneity $\left(\phi_{i}=15-25 \%\right)$ as compared to the one observed from the mCT images. While this discrepancy can not be explained based on our assessment of the uncertainty affecting mCT-computed porosity values, we cannot rule out that at this voxel size (i.e. the smallest achievable) the measurements might not be accurate enough. Nevertheless, for all three samples the correlation between the two set of data (192 measurements each) is significant, as confirmed from the values of the $R_{\mathrm{C}}$ coefficient reported in Table 1 . As anticipated in Section 3.3, watershed segmentation has been considered as an alternative approach to binarize the synchrotron images instead of hysteresis segmentation. In an analogous manner, results from this approach are presented in Figure 8 where, for plug P1, spatially distributed porosity values are plotted for the $2 \times 2$ (left-hand diagram) and $4 \times 4$ (right-hand diagram) schemes. In the figure, the black-filled symbols represent the solution obtained upon application of hysteresis thresholding ( $T_{\min }^{\mathrm{o}}=39$, as obtained from a best match to the medical CT data and shown in Figures 5 and 6), while the empty-filled symbols are results from watershed segmentation $\left(T_{\mathrm{min}}^{\mathrm{O}}=25\right)$. Accordingly, the effect of varying $T_{\min }$ on the watershed-segmented data is given by the results plotted as black lines $\left(T_{\min }=30-35\right)$. Two important observations can be made with respect to this figure. First, watershed segmentation suffers of the same problem affecting hysteresis thresholding, where a relatively small change in the parameter values used to binarize the image has a significant effect on the resulting porosity distribution of the rock. Secondly, and most importantly, an optimum set of threshold parameter values can be found also in this case that leads to a result that is practically identical to the one obtained by hysteresis thresholding (and, accordingly, by medical X-ray CT). In particular, the obtained concordance correlation coefficient takes a value of $R_{\mathrm{C}}=0.934,0.679$ and 0.346 when the image is coarsened to a voxel size of $(2 \times 2 \times 1),(1 \times 1 \times 1)$ and $(0.5 \times 0.5 \times 1) \mathrm{mm}^{3}$, respectively. The similarity between hysteresis- and watershed-segmented images shown in Figure 3 supports this conclusion and suggests that the approach presented here may be generally applicable to a wider range of locally-adaptive thresholding algorithms that have been developed to segment pores in different materials and rock types.

\section{Discussion}

In this study we have presented a quantitative assessment of X-ray tomography to probe the pore space of rocks across a continuum of relevant length-scales, i.e. from the pore- up to the core-scale. To this aim, rock samples have been imaged by medical X-ray CT and by synchrotron-based X-ray radiation, and a very good agreement has been found between the independently obtained porosity tomograms. The agreement has been quantitatively evaluated by means of a suitable statistical measure (the concordance correlation coefficient) and includes both average and spatially distributed porosity values at various spatial resolutions (i.e. voxel sizes). While for medical CT scanning standardization procedures have been developed over the years, one of the major issues reported in the literature with regards to the segmentation of micro-CT or synchrotron images is "the lack of ground truth, i.e. lack of knowledge of the optimal binarization result" [34]. In this context, the interesting corollary of this study is that a protocol has been established that exploits the comparison between the two techniques to inform the segmentation 
process. While previous studies have suggested that porosity alone is not sufficient to evaluate the quality of a segmented image [37], we like to emphasize that porosity is still the primary variable that can be obtained from binarized images, i.e. without the need for additional computational steps and their inherent assumptions. Furthermore, one of the benefits of the proposed approach is that for a given sample the spatial distribution of the porosity is compared in addition to its average value. The former is key to correctly predict other rock properties, such as the permeability or the capillary pressure curve, which indeed reflect distinct configurations of the pore space [47]. With the avowed intention of developing a protocol that enables a true multiscale imaging experiment, in this study we have restricted ourselves to Berea Sandstone, as this is probably the most widely studied (and imaged) rock type. While we have shown that also for a "standard" rock the image analysis process is far from being trivial, we also anticipate that the proposed workflow may be limited to rocks of similar microstructure. In the case of Berea Sandstone the latter is characterised by a fairly homogeneous (and monomineralic) composition $\left(>93 \% \mathrm{SiO}_{2}\right)$, the absence of micropores (i.e. pores below the resolution of the acquired images) and a significant fraction of pore space (15-25\%). In this context, future efforts should be directed towards the extension of the proposed approach to other (more complex) porous materials, such as carbonate rocks or soil samples, and to the evaluation of other properties, such as the permeability of the rock.

\subsection{The sensitivity of porosity to thresholding}

As a qualitative appraisal of the sensitivity of porosity to thresholding, reconstructed cuboids of the same sub-volume within plug P1 are shown in Figure 9 that have been obtained with different values of the parameter $T_{\min }$, namely 39 ( $\left.T_{\text {min }}^{\mathrm{o}}\right), 40,43,45$ (hysteresis thresholding). In the bottom panel of the figure, the corresponding differences $\left(T_{\mathrm{min}}^{\mathrm{o}}-T_{\mathrm{min}}\right)$ are represented. By naked eye, differences can hardly be seen when the $3 \mathrm{D}$ reconstructions shown in the top panel of the figure are considered. The differences within each single slice (i.e. stacked image) are subtle, as it can be appreciated by looking at the side of the cuboid that has been grey-faced for this purpose (bottom panel). However, when the entire volume of the cuboid is considered, a cloud of points appears and grows as one moves farther from the optimal threshold level. As discussed in the next paragraph, the cumulative effects of these differences has a significant impact on the computed porosity of the sample. With this regard, the identification of the optimum threshold level based on individual (sets of) 2D images represents a deceptive approach, which may lead to a significant underestimation of the effects caused by the selection of the wrong threshold level.

Figure 10 shows the correlation between porosities computed from mCT- and $\mu \mathrm{CT}$ images plotted in histogram form as a function of the thresholding parameter used for segmenting the $\mu \mathrm{CT}$ images with the hysteresis method. With 'correlation' we refer here to the mean value of the concordance correlation coefficients obtained when comparing images at three different voxel sizes. The top panel of the figure additionally shows the corresponding error in the average sample porosity (relative to the $\mathrm{mCT}$ values). It is evident from the plots that an optimum value for the thresholding level can indeed be identified (darker bars) and that the effects of the 
thresholding parameter on the porosity are not negligible, with relative errors up to $10 \%$. Two comments are worth making with respect to this point. First, for a given sample a relative error of about $10 \%$ is considered significant, because porosity varies of the same amount when different samples are compared (see porosity values in Table 1). It follows that a poor binarization of the $\mu \mathrm{CT}$ images would preclude the quantification of porosity heterogeneity, the latter being the reason why non-invasive imaging are applied in the first place. Secondly, such errors are not likely to be reduced by the adoption of a different segmentation algorithm than the one primarily used in this study. In fact, while the variety of available methods have been shown to provide as many different results, most of them require a significant degree of supervision and the choice of the given threshold value is inherently operator-biased $[34,36,37]$. Most importantly, we have shown in this study that the adoption of two different thresholding algorithms (i.e. watershed and hysteresis segmentation) applied to the same sample (image) provides almost identical results upon proper selection of thresholding parameters' values. As a matter of fact, we argue that the point at issue is not the choice of the best segmentation method, but rather the development of protocols that objectively validate the results from any of these approaches. We have proposed in this study a practical method to achieve this.

\subsection{A true multi-scale imaging experiment}

The characterization of the complex pore space of rocks starts from the analysis of reservoir core samples and the ability to quantitatively link observations across orders of length-scales is a key step in this process. For instance, up to six registered resolutions may be required to properly capture the heterogeneity of carbonate rocks, thus including micro-structures that control pore connectivity as well as larger features that define permeability variations at the $\mathrm{cm}$ scale $[23,24]$. However, while the imaging of whole cores by $\mathrm{mCT}$ is limited to a resolution of a few $\mathrm{mm}$ (i.e. corresponding to a voxel volume containing already hundreds if not thousands of grains), an issue that limits the use of high-resolution $\mu \mathrm{CT}$ images is the up-scaling of the computed properties to the core-scale [23]. As shown in the present study, the proper coupling between these two tomographic techniques has the potential to fill this gap. In fact, once calibrated against $\mathrm{mCT}$ data, $\mu \mathrm{CT}$ images allow extending the analysis of the pore space down to the micron level thanks to their enhanced resolution, thus enabling a true multi-scale experiment. This concept is presented in Figure 11 for a Berea Sandstone core sample. In the figure, the porosity is plotted of sub-core elements with volumes that span over more than eight orders of magnitudes, i.e. from a single grain up to the whole sample that contains $\sim 10^{8}$ grains. Solid lines are porosity values computed from $\mu \mathrm{CT}$ images of the three 6 -mm plug samples, while symbols represent results obtained from $\mathrm{mCT}$ images of the intact 5 -cm core sample. The former are presented in the form of REV curves [48], i.e. by calculating the porosity of an increasingly larger rectangular cuboid starting from a voxel centered in the pore space up to the size of the imaged sample.

As expected, porosity initially decreases with increasing cuboid volume, due to the incorporation of an increasingly large volumetric fraction of the solid component of the rock. This is followed by a region $\left(0.01-5 \mathrm{~mm}^{3}\right)$ with relatively large 
fluctuations in the computed porosity, before leveling off at a value that differs for each sample considered. The latter obviously reflects the inhomogeneity of the rock sample at this scale (see values in Table 1). Interestingly, a constant porosity value is attained for each sample beyond a value of about $5 \mathrm{~mm}^{3}$, thus suggesting that this volume (the so-called Representative Elementary Volume, REV) includes enough pores and grains such that their random orientation becomes insignificant. In other words, while a $5 \mathrm{~mm}^{3}$ element would be sufficient to represent any imaged small plug, it would still not capture the spatial variability in the porosity that characterizes the whole rock sample. This conclusion is supported by the independent estimation of porosity heterogeneity from the mCT images; the latter is represented by the "error" bars associated to each symbol in the figure that have been estimated from the standard deviation of the distribution of porosity values observed for a given voxel size by considering the whole core sample (with a diameter of $5 \mathrm{~cm}$ and length of $8.1 \mathrm{~cm}$ ). The quantitative agreement between the scatter of the solid lines and the size of the error bars is rather striking. Additionally, the shaded region highlights the range of voxel volumes where a direct comparison between the two techniques can be made and that has been exploited in the present study to inform the binarization of the $\mu \mathrm{CT}$ images. This has in turns allowed populating a larger image captured at a poorer resolution with detailed information on the pore space of the rock at a resolution of a few microns. One of the first benefits of this type of analysis is that it provides the data-set to investigate the impact of heterogeneity at multiple scales, thus including various rock properties and their association with flow. Accordingly, such comprehensive data-set can in turn be exploited for the validation and/or refinement of suitable up-scaling approaches that capture the inherently complex heterogeneity of rocks.

\section{Concluding remarks}

In this study, medical X-ray CT and synchrotron-based X-ray radiation have been applied to image and quantify the porosity of three Berea Sandstone samples. The experiments have been carried out such that porosity measurements on the same volume of sample are compared in terms of both averaged and spatially distributed properties. The main conclusions from this study are summarized as follows:

- A excellent agreement is obtained between porosity values measured by the two independent techniques for an imaged volume of $(4 \times 4 \times 1) \mathrm{mm}^{3}$ and voxel sizes down to $(1 \times 1 \times 1) \mathrm{mm}^{3}$.

- Methods are presented to reduce the (inherent) noise associated with the X-ray tomograms and to account for uncertainties related the physical sub-sampling of the plugs used for high-resolution imaging. An appropriate quantitative measure (i.e. the concordance correlation coefficient) has been used to evaluate the agreement between the two techniques.

- The established protocol exploits the comparison between the two techniques to objectively inform the segmentation process (i.e. the threshold level) required for the analysis of micro-CT tomograms that would otherwise be prone to operator bias.

- The applicability of the proposed workflow is demonstrated with two widely applied locally adaptive thresholding algorithms, namely hysteresis (or bilevel) and watershed segmentation. 
- The arbitrary selection of the threshold level may lead to relative errors up to $10 \%$ in the estimated average porosity of a given sample with a volume of $\sim 16 \mathrm{~mm}^{3}$. This error is considered significant, because porosity varies of the same amount when different samples are compared at this scale.

- The ability to quantify porosity with such detail enables creating a direct link between pore-scale structures (observed by means synchrotron-based radiation or micro X-ray CT) with sub-core scale patterns (observed on rock cores by means of medical X-ray CT). Efforts in this direction are justified by the need of developing experimental protocols to probe the pore space of rocks across the continuum of relevant length-scales (true multi-scale imaging).

\section{Appendix}

\section{A.1 Quantification of the mCT noise}

The use of tomograms obtained with a medical CT scanner on a voxel-by-voxel basis requires a careful appraisal of the uncertainty associated with readings at such high resolutions. A practical way for achieving this is to subtract images of scans taken at identical locations (e.g. two consecutive scans of the same slice); as described in [13], the obtained differences follow a Normal distribution function that is centred around zero and that is characterised by a given standard deviation. The latter is a measure of the "CT noise". This analysis can be readily extended by (i) subtracting images that represent an average of multiple scans of the same slice and/or by (ii) subtracting images obtained at increasing voxel sizes. Both methods can in fact effectively reduce image noise. Results from such exercise are shown in Figure 12, where the "CT noise" is plotted as a function of the number of averaged scans $n$ (represented in terms of $1 / \sqrt{n}$ in the figure) for the three different voxel sizes considered in this study, namely $(0.5 \times 0.5 \times 1),(1 \times 1 \times 1)$ and $(2 \times 2 \times 1) \mathrm{mm}^{3}$. Upon application of classic rules of error propagation, it can be shown that [13]:

$$
\sigma_{\Delta_{n}}=\sqrt{2} \frac{\sigma_{\mathrm{vox}}}{\sqrt{n}}
$$

where $\sigma_{\Delta_{n}}$ (the "CT noise") is the standard deviation that represents the distribution of CT values obtained upon subtracting two images, which have been obtained from an average $n$ independent scans each, while $\sigma_{\text {vox }}$ is the uncertainty in CT units associated to a single voxel. In Figure 12, the symbols are the experimentally obtained $\sigma_{\Delta_{n}}$ from a set of 20 consecutive scans of the same slice of the Berea Sandstone sample used in this study, while the lines represent the behaviour predicted by Eq.A-1. Accordingly, $\sigma_{\text {vox }}$ takes a value of 18.4, 15.0 and $8.8 \mathrm{HU}$ for a voxel of size $(0.5 \times 0.5 \times 1),(1 \times 1 \times 1)$ and $(2 \times 2 \times 1) \mathrm{mm}^{3}$, respectively. When 20 consecutive scans are taken, this uncertainty reduces to $4.1,3.3$ and $2.0 \mathrm{HU}$, respectively. In an analogous manner, it can be shown that porosity values predicted from Eq. 1 are affected by an uncertainty $\sigma_{\phi, n}$ :

$$
\sigma_{\phi_{n}}=\sqrt{2} \frac{\sigma_{\mathrm{vox}}}{\sqrt{n} 1000}=\frac{\sigma_{\Delta_{n}}}{1000}
$$

where $\phi_{n}$ represents a voxel porosity obtained upon combination of dry and wet images, which have been obtained from an average $n$ independent scans each [13]. 
Accordingly, porosity uncertainties are reported on the right-hand side y-axis in Figure 12 and the corresponding 2D porosity maps are shown in Figure 2.

Acknowledgements Dr. Claudio Madonna is grateful to Prof. Sally Benson, Department of Energy Resources Engineering, Stanford University (Stanford, USA) for supporting his research visit. Special thanks go to Dr. Jonathan Ajo-Franklin and Dr. Marco Votolini, Lawrence Berkeley National Laboratory (Berkeley, USA) for the acquisition and initial reconstructing of the synchrotron images.

\section{References}

1. D. Wildenschild, A.P. Sheppard, Adv. Water Res. 51, 217 (2013)

2. F. Fusseis, X. Xiao, C. Schrank, F. De Carlo, J. Struct. Geol. 65, 1 (2014)

3. S. Wellington, H. Vinegar, J. Pet. Technol. 39, 885 (1987)

4. E.M. Withjack, SPE Formation Eval. 3, 696 (1988)

5. V. Cnudde, M. Boone, Earth Sci. Rev. 123, 1 (2013)

6. D. Silin, L. Tomutsa, S. Benson, T. Patzek, Transport Porous Med. 86(2), 495 (2011)

7. M. Andrew, B. Bijeljic, M.J. Blunt, Geophys. Res. Lett. 40(15), 3915 (2013)

8. A.L. Herring, L. Andersson, D. Newell, J. Carey, D. Wildenschild, Int. J. Greenhouse Gas Control 25, 93 (2014)

9. S.C.M. Krevor, R. Pini, L. Zuo, S.M. Benson, Water Resour. Res. 48(2), W02532 (2012)

10. S. Berg, S. Oedai, H. Ott, Int. J. Greenhouse Gas Control 12, 478 (2013)

11. R. Pini, S.M. Benson, Water Resour. Res. 49(6), 3516 (2013)

12. M. Akbarabadi, M. Piri, Adv. Water Res. 52, 190 (2013)

13. R. Pini, S.C.M. Krevor, S.M. Benson, Adv. Water Res. 38, 48 (2012)

14. R.H. Brooks, A.T. Corey, Hydrology Paper 3, 1 (1964)

15. W. Murphy, J. Roberts, D. Yale, K. Winkler, Geophys. Res. Lett. 11(8), 697 (1984)

16. K.A. Klise, V.C. Tidwell, S.A. McKenna, Adv. Water Res. 31(12), 1731 (2008)

17. J.C. Perrin, S. Benson, Transport Porous Med. 82(1), 93 (2010)

18. J.Q. Shi, Z. Xue, S. Durucan, Int. J. Greenhouse Gas Control 5(1), 75 (2011)

19. B.L. Alemu, E. Aker, M. Soldal, Ø. Johnsen, P. Aagaard, Geophys. Prospect. 61(1), 235 (2013)

20. S. Krevor, R. Pini, B. Li, S. Benson, Geophys. Res. Lett. 38, L15401 (2011)

21. R. Pini, S.M. Benson, Geophys. Res. Lett. 40(15), 3903 (2013)

22. T. Tran, J. Hydrol. 183(1), 37 (1996)

23. A.D. Grader, A.B. Clark, T. Al-Dayyani, A. Nur, in Proceedings of the International Symposium of the Society of Core Analysts (2009), pp. Noordwijk aan Zee, The Netherlands, 27-30 September

24. R.M. Sok, T. Varslot, A. Ghous, S. Latham, A.P. Sheppard, M.A. Knackstedt, Petrophysics 51(6), 379 (2010)

25. P. Lai, K. Moulton, S. Krevor, Chem. Geol. 411, 260 (2015)

26. R. Pini, Langmuir 30(37), 10984 (2014)

27. S. Akin, A.R. Kovscek, in Applications of X-ray Computed Tomography in the Geosciences, vol. 215, ed. by F. Mees, R. Swennen, M. Van Geet, P. Jacobs (Geological Society, London, 2003), pp. 23-38

28. H. Ott, K. de Kloe, M. van Bakel, F. Vos, A. van Pelt, P. Legerstee, A. Bauer, K. Eide, A. van der Linden, S. Berg, Rev. Sci. Instr. 83(8), 084501 (2012)

29. Y. Zhang, O. Nishizawa, T. Kiyama, S. Chiyonobu, Z. Xue, Geophys. J. Int. 197(3), 1789 (2014)

30. B. Vega, A. Dutta, A.R. Kovscek, Transport Porous Med. 101(1), 81 (2014)

31. E.J. Peters, W.D. Hardham, J. Pet. Sci. Eng. 4(2), 155 (1990)

32. S. Ganapathy, D.G. Wreath, M.T. Lim, B.A. Rouse, G.A. Pope, K. Sepehrnoori, SPE Formation Eval. 8(4), 273 (1993)

33. M. Honarpour, A. Cullick, N. Saad, N. Humphreys, J. Pet. Technol. 47, 980 (1995)

34. P. Iassonov, T. Gebrenegus, M. Tuller, Water Resour. Res. 45(9) (2009)

35. H. Andrä, N. Combaret, J. Dvorkin, E. Glatt, J. Han, M. Kabel, Y. Keehm, F. Krzikalla, M. Lee, C. Madonna, Comput. Geosci. 50, 25 (2013) 
36. P.C. Baveye, M. Laba, W. Otten, L. Bouckaert, P. Dello Sterpaio, R.R. Goswami, D. Grinev, A. Houston, Y. Hu, J. Liu, Geoderma 157(1), 51 (2010)

37. L. Leu, S. Berg, F. Enzmann, R. Armstrong, M. Kersten, Transport Porous Med. 105(2), $451(2014)$

38. M.L. Porter, D. Wildenschild, Comput. Geosci. 14(1), 15 (2010)

39. C.E. Shannon, Proc. IRE 37(1), 10 (1949)

40. A. Buades, B. Coll, J.M. Morel, IPOL J. 1, http://dx.doi.org/10.5201/ipol.2011.bcm_nlm (2011)

41. H. Vogel, A. Kretzschmar, Geoderma 73(1), 23 (1996)

42. L. Vincent, P. Soille, IEEE Trans. Pattern Anal. Mach. Intell. 6, 583 (1991)

43. S. Schlüter, A. Sheppard, K. Brown, D. Wildenschild, Water Resour. Res. 50(4), 3615 (2014)

44. G. Kerckhofs, J. Schrooten, T. Van Cleynenbreugel, S.V. Lomov, M. Wevers, Rev. Sci. Instr. 79(1), $013711(2008)$

45. N.J. Cox, Geomorphology 76(3), 332 (2006)

46. J.R. Taylor, An introduction to error analysis, 2nd edn. (University Science Books, 1997)

47. M. Chaouche, N. Rakotomalala, D. Salin, B. Xu, Y. Yortsos, Chem. Eng. Sci. 49(15), 2447 (1994)

48. J. Bear, Dynamics of Fluids in Porous Media (Dover Publications, Inc., 1988) 


\section{Tables}

Table 1 Average porosity values, $\phi$, of the three samples used in this study that have been computed from mCT and $\mu \mathrm{CT}$ images. The concordance correlation coefficient, $R_{\mathrm{C}}$, quantifies the agreement between the two techniques at different spatial resolutions, i.e. for a $(4 \times 4 \times 1) \mathrm{mm}^{3}$ cuboid containing $(2 \times 2),(4 \times 4)$ and $(8 \times 8)$ voxels.

\begin{tabular}{clllll}
\hline Sample & Medical CT & Synchrotron & Agreement & \\
& Porosity & Porosity & Concordance correlation & \\
& $\phi[\%]$ & $\phi[\%]$ & $R_{\mathrm{C}}(2 \times 2)$ & $R_{\mathrm{C}}(4 \times 4)$ & $R_{\mathrm{C}}(8 \times 8)$ \\
P1 & $20.7(2)$ & $20.7(2)$ & 0.939 & 0.681 & 0.350 \\
P2 & $19.9(6)$ & $19.8(7)$ & 0.672 & 0.691 & 0.251 \\
P3 & $18.9(3)$ & $19.0(3)$ & 0.813 & 0.532 & 0.308 \\
\hline
\end{tabular}




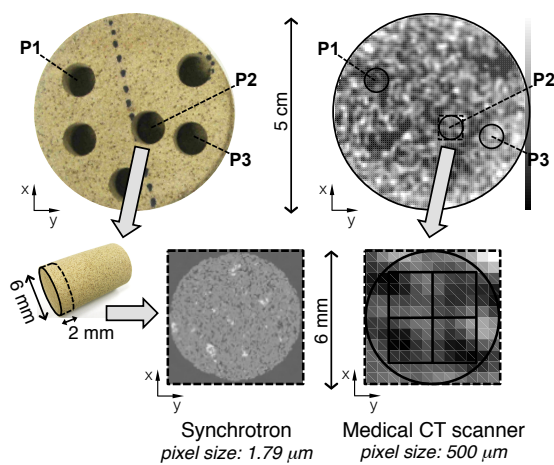

Fig. 1 The workflow of the multi-scale imaging experiment presented in this study. Upper left corner: digital photograph of the inlet face of the Berea Sandstone sample (diameter: $5 \mathrm{~cm}$ ) taken after the coring of smaller sub-samples (diameter: $6 \mathrm{~mm}$ ). Upper right corner: the corresponding $1 \mathrm{~mm}$-thick dry-scan obtained by medical X-ray CT scanning prior to resampling (gray scale in Hounsfield Units, voxel size: $\left.(0.5 \times 0.5 \times 1) \mathrm{mm}^{3}\right)$. Bottom right corner: close-up for sub-sample P2 at the same voxel size. Bottom left corner: a representative slice of sample P2 imaged with synchrotron-based X-ray radiation $(1.79 \mu \mathrm{m} / \mathrm{pixel})$. 


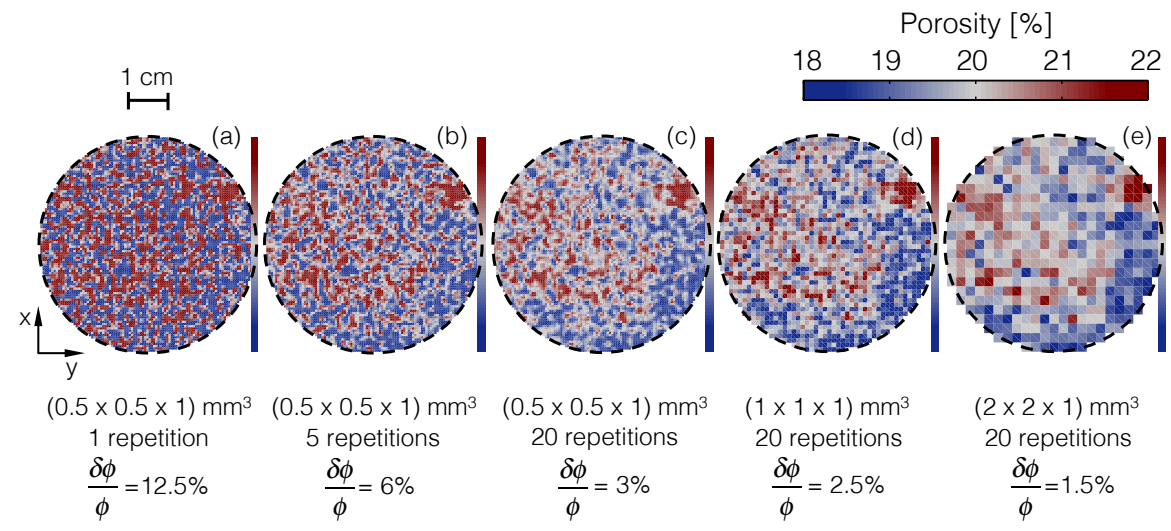

Fig. 2 Reduction of the noise affecting the mCT scans and represented here in terms of $2 \mathrm{D}$ porosity maps of the $1 \mathrm{~mm}$ thick inlet slice of the mother rock sample. Images (a), (b) and (c) represent the same slice that has been obtained at the scanner's original resolution corresponding to a voxel size of $(0.5 \times 0.5 \times 1) \mathrm{mm}^{3}$ and upon averaging 1,5 and 20 repeated scans, respectively. Images (d) and (e) are obtained upon coarsening the grid of the original scans with a $(2 \times 2)$ and a $(4 \times 4)$ scheme, respectively. The corresponding voxel sizes are given below each image together with the relative uncertainties in the computed voxel porosity $(\delta \phi / \phi)$. Note that irrespectively of the protocol applied, the average slice porosity is known with a relative precision of $0.2 \%$. 
A. Raw image

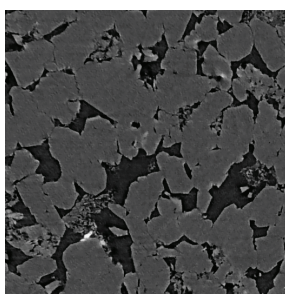

Matrix
B. Filtered image (NLM) C. Hysteresis thresh.
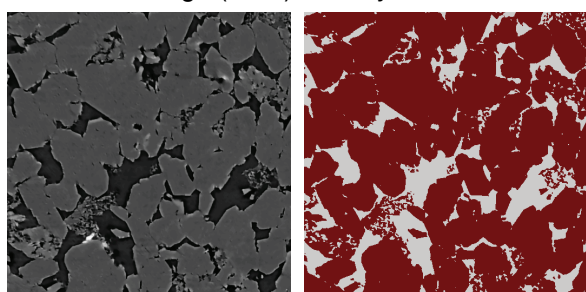

Pores
Watershed thresh.

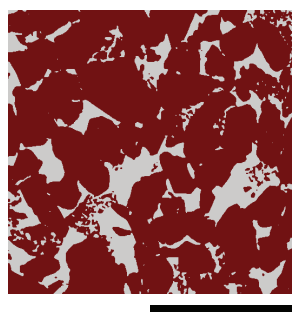

Fig. 3 Overview of the image processing steps applied to the $\mu \mathrm{CT}$ tomograms, as shown by means of a $(1 \times 1) \mathrm{mm}^{2}$ close-up figure of the raw (A), filtered $(B)$ and segmented $(C$ and D) image. Images are first passed through a non-local mean denoising filter (NLM) and then binarized by means of either bilevel (hysteresis) or watershed thresholding. See manuscript text for details regarding the applied parameters' values. 


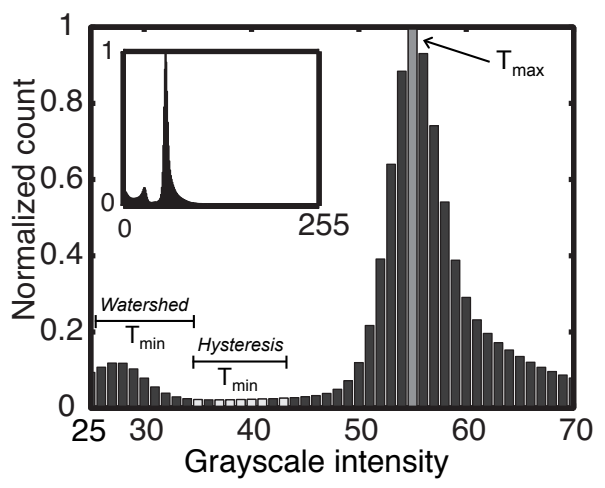

Fig. 4 Histogram of the grey-level intensity distribution of the 8-bit image obtained by synchrotron-based X-ray radiation and representing a $(2 \times 2 \times 2) \mathrm{mm}^{3}$ volume at a resolution of $1.79 \mu \mathrm{m} /$ pixel. For both the hysteresis and watershed thresholding, $T_{\max }$ is set to a value of 55 , while $T_{\min }$ is used as a fitting parameter, so as to obtain an optimum match with independent measurements by medical X-ray CT (ranges shown in the figure by the horizontal bars). 

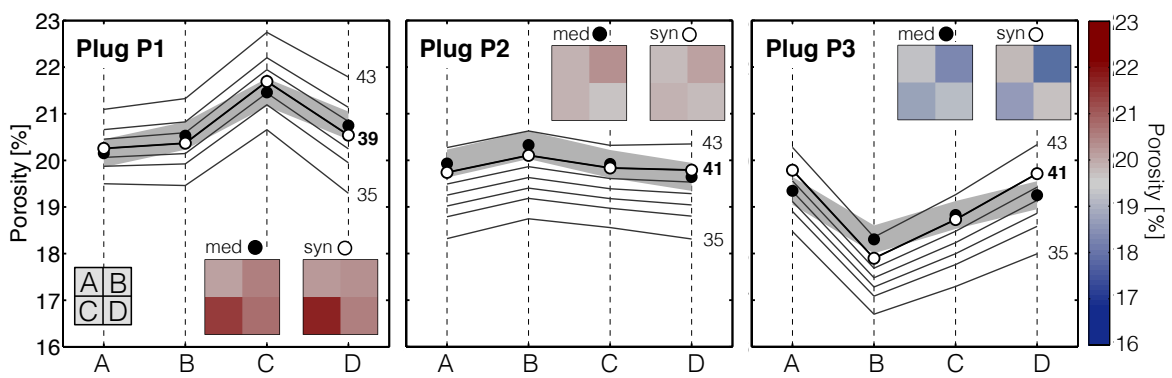

Fig. 5 Comparison between porosity values obtained from mCT- (filled black circles with an uncertainty of $\pm 0.3 \%$ abs. represented by the grey-shaded region) and $\mu \mathrm{CT}$-images (black empty symbols) for samples P1, P2 and P3. Porosity values correspond to four different subquadrants (A, B, C and D) in each sample and are additionally shown in the colored insets in the form of 2D maps for both medical- ('med') and synchrotron-based tomography ('syn'). The black lines are results obtained by varying the threshold level $\left(T_{\min }\right)$ for segmenting the synchrotron images. Sample size: $(4 \times 4 \times 1) \mathrm{mm}^{3}$, voxel size: $(2 \times 2 \times 1) \mathrm{mm}^{3}$. 


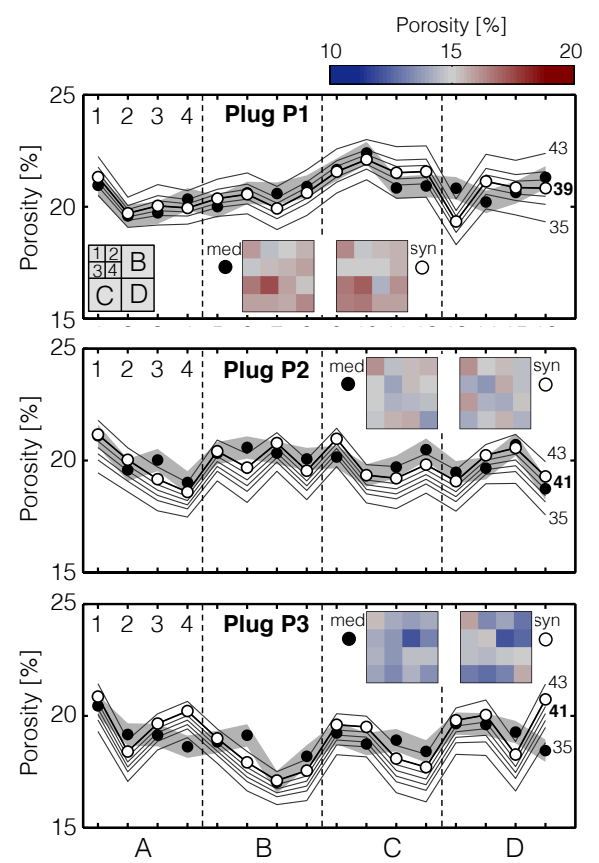

Fig. 6 Comparison between mCT- (filled black circles with an uncertainty of $\pm 0.5 \%$ represented by the grey-shaded region) and $\mu \mathrm{CT}$-porosity values (black empty symbols) for samples P1, P2 and P3. The results represent sixteen different sub-quadrants (A1, A2, A3, A4, B1, $\mathrm{B} 2$, etc.) in each sample and are additionally shown in the colored insets in the form of $2 \mathrm{D}$ maps for both medical- ('med') and synchrotron-based tomography ('syn'). The black lines are results obtained by varying the threshold level $\left(T_{\min }\right)$ for segmenting the synchrotron images. Sample size: $(4 \times 4 \times 1) \mathrm{mm}^{3}$, voxel size: $(1 \times 1 \times 1) \mathrm{mm}^{3}$. 

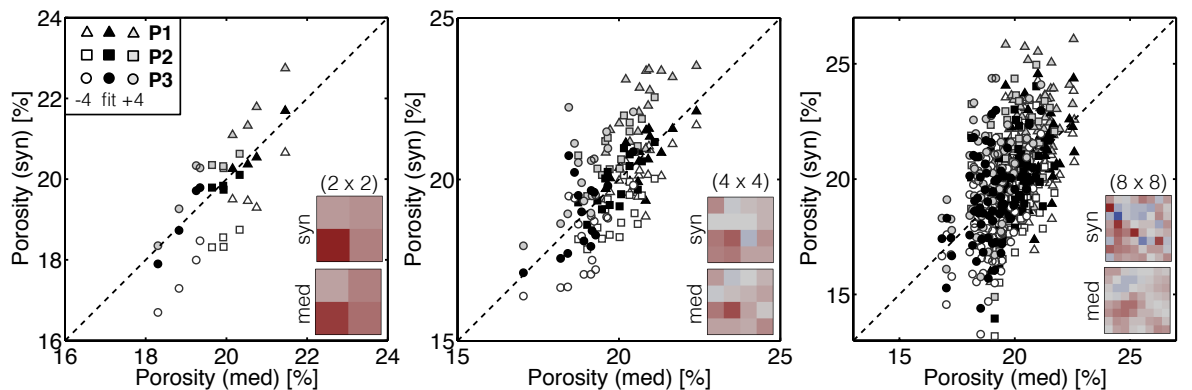

Fig. 7 Correlation diagrams of the porosity values obtained from $\mu \mathrm{CT}$ images plotted as a function of the corresponding results obtained from $\mathrm{mCT}$ images for samples P1 (triangles), P2 (squares) and P3 (circles). The three diagrams represent comparisons made at different voxel sizes, namely $(2 \times 2 \times 1),(1 \times 1 \times 1)$ and $(0.5 \times 0.5 \times 1) \mathrm{mm}^{3}$ (from left to right), and include the optimum fit (black filled symbols), together with solutions obtained using a larger (greyfilled symbols) and a smaller value (empty symbols) of the threshold level $\left(T_{\min }\right)$ used for segmenting the $\mu \mathrm{CT}$ images. A perfect correlation is represented by the dashed black identity line, while the colored insets are 2D visual representation of sample P1. 

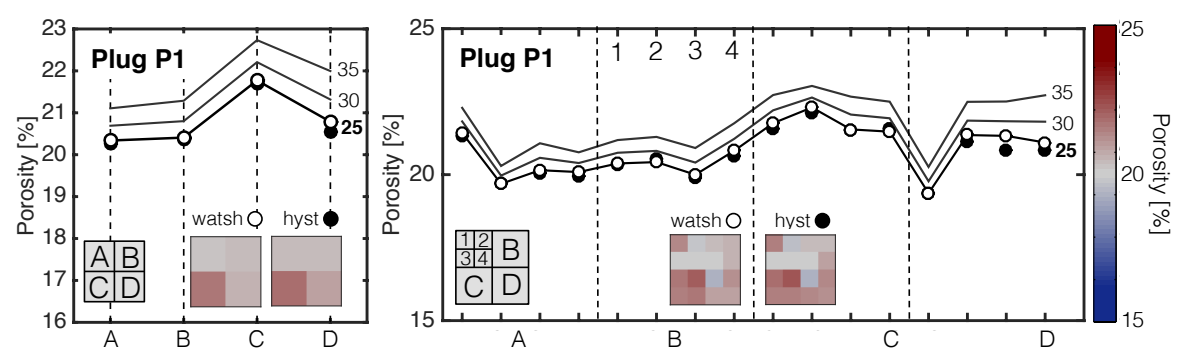

Fig. 8 Comparison between $\mu \mathrm{CT}$-porosity values obtained for sample P1 upon application of the hysteresis (black filled symbols, $T_{\min }=39$ ) and watershed segmentation methods. Results obtained from the latter include the optimum fit (empty filled symbols, $T_{\min }=25$ ), together with solutions obtained by varying the threshold level (black lines, $T_{\min }=30,35$ ). Results are shown for two voxel sizes, namely $(2 \times 2 \times 1) \mathrm{mm}^{3}$ (left diagram) and $(1 \times 1 \times 1) \mathrm{mm}^{3}($ right diagram). The coloured insets added to each diagram provide a $2 \mathrm{D}$ representation of the same data. 

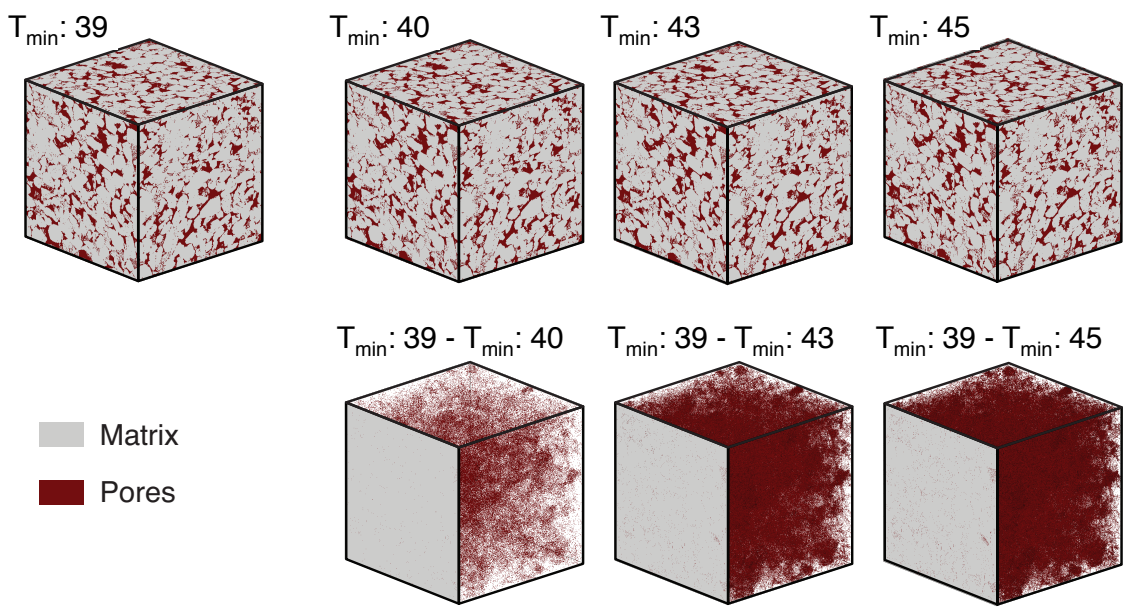

Fig. 9 Segmentation of the synchrotron images. Reconstructed cuboids representing the same sub-volume within the Berea sandstone plug P1 obtained with different values of the threshold parameter $T_{\min }$, namely $39\left(T_{\mathrm{min}}^{\mathrm{o}}\right), 40,43,45$. These are shown in the upper panel of the figure, while the corresponding differences $\left(T_{\min }^{\circ}-T_{\min }\right)$ are shown in the bottom panel. 


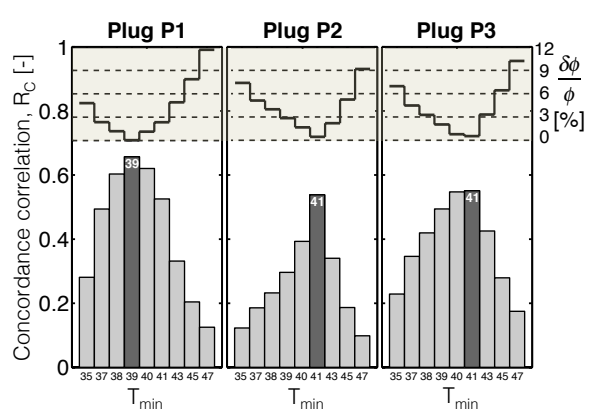

Fig. 10 Histograms showing the obtained values for the mean concordance correlation, $R_{\mathrm{C}}$, between porosities obtained from medical X-ray CT and synchrotron-based radiation as a function of the threshold level $\left(T_{\min }\right)$ used for segmenting the synchrotron image for sample P1, P2 and P3. Plotted values represent the mean of all three coarsening schemes, namely $(2 \times 2 \times 1),(1 \times 1 \times 1)$ and $(0.5 \times 0.5 \times 1) \mathrm{mm}^{3}$. Best fit results (bars with darker color) are reported in Table 1 together with values for each scheme. In the top panel of the figure is shown the error in the total sample porosity obtained from $\mu \mathrm{CT}$ images relative to $\mathrm{mCT}$ values. 


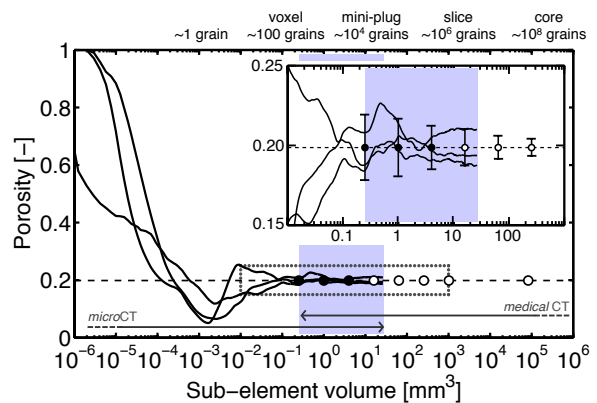

Fig. 11 Quantification of the porosity of a Berea Sandstone core $(5 \mathrm{~cm}$ in diameter and $8.1 \mathrm{~cm}$ long) over 12 orders of magnitudes. Solid lines are results from the segmented $\mu \mathrm{CT}$ images of three distinct samples (P1, P2 and P3) with volume $(4 \times 4 \times 2) \mathrm{mm}^{3}$; the porosity is calculated for an increasingly larger rectangular cuboid starting from a voxel centered in the pore space. Symbols are results from mCT images of the whole core sample consisting of eighty-one $1 \mathrm{~mm}$ thick slices; each symbol is associated to a different coarsening scheme applied to the images and the 'error' bars represent the spatial variability of the calculated porosity (the length of each side of the bar is twice the standard deviation of the distribution of porosity values). The filled symbols are voxel-porosity values calculated within a single slice that has been obtained from the average of 20 repeated scans taken at the same location, so as to reduce measurement uncertainty (see Figure 2). 


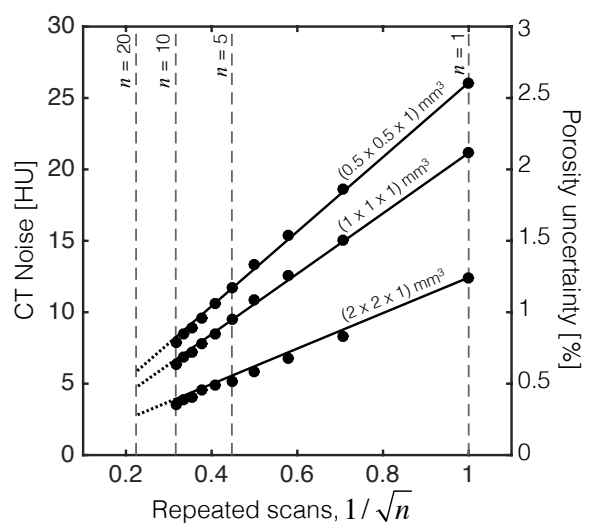

Fig. 12 Analysis of the noise affecting tomograms acquired with a medical CT scanner at various voxel sizes, namely $(2 \times 2 \times 1),(1 \times 1 \times 1)$ and $(0.5 \times 0.5 \times 1) \mathrm{mm}^{3}$. The CT noise is defined as the standard deviation, $\sigma_{\Delta_{n}}$, that represents the distribution of CT values obtained upon subtracting two images, which have been obtained from an average $n$ independent scans each.

Experimentally determined values are given by the symbols, while the lines are predictions from Eqs.A-1 (left-hand y-axis) and A-2 (right-hand y-axis). X-ray CT images were acquired on a General Electric Hi-Speed CT/i X-ray computed tomography scanner by selecting a tube current of $200 \mathrm{~mA}$ and an energy level of the radiation of $120 \mathrm{keV}$. 